\title{
Gli aspetti più importanti del problema del sistema motorio extrapiramidale
}

Resumo da conferencia do prof. G. Mingazzini na Sociedade Arnaldo Vieira de Carvalho, feito pelo autor para a "Revista de Medicina"

Drima di parlare di questo spinoso argomento, la cui discussione, iniziata appena cinque lustri or sono, ha giá affaticato le menti dei più illustri neuropatologi del mondo, è necessario premettere una definizione precisa.

Per Sistema Motorio Extrapiramidale si intende un complesso di formazioni anatomiche, le quali inviano, in parte al midollo allungato e in parte alle corna anteriori del midollo spinale, un sistema di fibre, che rinforzano la dinamica esplicantesi dai neuroni della via piramidale.

Le formazioni costitutive di questo sistema non sono ancora del tutto bene accertate, specialmente per quanto concerne il loro decorso e, sopratutto, la loro esatta terminazione.

Si può ad ogni modo asserire che il principale contingente è rappresentato dal nucleo lenticolare, e più propriamente dal globus palli$d u s$, e dall'associazione di due gangli, il caudatus e il putamen, conosciuti sotto il nome complessivo di striatum.

Un altro ganglio che, specialmente negli animali, concorre a rinforzare il sistema, è il nucleo rosso, il quale emette un fascio, il fasciculus rubro-spinalis, che decorre al dinanzi del fascio piramidale crociato.

Pari' per importanza ai due precedenti, come componente del sistema extapiramidale, è il nucleo di Deiters, destinato alle funzioni vestibolari, i cui rami discendenti vanno a disporsi nel midollo spinale, lungo il margine ventrale del cordone anteriore.

Infine va ricordata la substantia nigra, la quale nell'uomo assume un'importanza assai maggiore che negli altri mammiferi supe- 
riori. Dalle sue cellule infatti emanano cilindrassili che si accollano, almeno in parte, al fascio piramidale del pes pedunculi.

Incerti sono $\mathrm{i}$ contributi che alcuni assegnano anche al corpo subtalamico e perfino ai gangli del tuber cinereum.

Mi limiteró a parlare del più importante dei gangli, cioè del sistema strio-pallidale, essendo a questo proposito le nostre conoscenze abbastanza esatte.

La fisiologia ha cercato per lungo tempo di studiare gli effetti consecutivi all'irritazione o alla distruzione del lenticolare; ma inutilmente; la posizione di questo ganglio è infatti tale, che non è possibile aggredirlo sia dall'alto che dai lati, senza ledere contemporaneamente giri o solchi dotati di funzioni essenziali.

Tutte le nostre conoscenze sulla fisiologia di questo ganglio sono dovute alle alterazioni che frequentemente lo colpicono. E poichè nell'ultimo settennio l'umanità ha avuto la sventura di essere inondata da una fatale malattia, quale l'encefalite epidemica, che predilige il lenticolare, si comprende come gli anatomopatologi e $\mathrm{i}$ cultori di neurologia abbiano trovato larga messe per i relativi loro studi.

$\mathrm{Ma}$ non solo l'anatomia patologica, ma anchhe l'embriologia, e propriamente lo studio della mielinizzazione dei due segmenti del lenticolare, ci hanno dato risultati abbastanza sóddisfacenti.

Senza entrare in minuzie istologiche, diró come le lesioni del pallidus producano una sindrome totalmente diversa da quella dello striatum. Nelle prime si svolge un complesso sintomatico, caratteristico della sindrome di Parkinson, costituito da un aumento notevole del tono, associato a lentezza dei movimenti, tanto da giungere talvolta a una vera e propria acinesia. Nelle lesioni dello striatum si osserva invece l'insorgere di una molteplicità di movimenti, a carattere quasi automatico, con carattere ad es. di difesa, di aggressione, di prensione e così via. Oltre a ciò in antitesi a quanto si osserva nelle lesioni del pallidus l'esame dei movimenti passivi rivela una spiccata ipotonia. Un paradigma di questo quadro clinico si ha nella chorea.

La conclusione che logicamente si è tratta da tali osservazioni si è che il pallidus è la sede dei movimenti automatici polimorfi ereditari, quali noi vediamo nel neonato; lo striatum invece ha l'ufficio di correggere e di coordinare i movimenti stessi, e di aggiungere quella funzione tònica della quale il bambino è completamente privo.

Hanno dunque ragione gli embriologi, quando danno il nome di paleostriatum al pallidus, e di neostriatum al putamen e al caudatus.

E' questa la ragione per la quale la mielinizzazione del pallidus comincia nei primi mesi della vita extrauterina, mentre quella del putamen si svolge assai più tardi, quando il bambino comincia a disciplinare i suoi scomposti movimenti. 
Quali siano le vie per mezzo delle quali neo e paleostriatum inviano i loro impulsi ai fasci piramidali volontari, è problema tutt'altro che risolto. Gli schemi che alcuni autori di grande fama, come Wilson e altri, hanno proposto, sono, se non arbitrari, almeno incerti.

Io che mi sono occupato a lungo, con ricerche originali e personali, di questo argomento, credo di poter affermare con sicurezza che i fasci provenienti dal lenticolare si accollano alla via piramidale all'altezza della capsula interna.

Certamente molte e importantissime questioni sul problema del sistema motorio extrapiramidale rimangono per ora insolute, e molti anni occorreranno prima che sull'argomento estremamente complesso si possa fare un po' più di luce.

Laboratoire des Produits "Usines du Rhone" 21, RUE JEAN GOUJON - PARIS

\section{A CETYLARSAN}

(OXYACETYLAMINA PHENYLARSINATO DE DIETHYLAMINA) TRATAMENTO DA SYPHILIS

1. Actividade Igual a 3. RAPIDEZ DE AC ÇÃO DOS 'ARSENO-BENZÓES

$4 .^{\circ}$ Facilidade $D E$ EMPREgo POR INJECÇÕES INTRAMUS-

2. Tolerancia Perfeita CULARES INDOLARES

Amostras e litteratura aos Medicos mediante pedidos AOS CONCESSIONARIOS ESCLUSIVOS

Companhia Chimica Rhodia Brasileira OAIXA POATAL 1329

S. PA U L O 